\title{
Cultura escolar y cultura juvenil: La (re)construcción simbólica del espacio escolar en la mutación cultural
}

\author{
Walter Molina, Mario Sandoval ${ }^{*}$
}

\begin{abstract}
Resumen
En términos amplios, nuestra reflexión se ubica en el contexto de las transformaciones sociales, económicas, políticas y culturales que viven las sociedades contemporáneas. Estas transformaciones se han perfilado como uno de los principales fenómenos en el inicio del Siglo XXI y han sido conceptualizadas de diversos modos: "sociedad de la información", "sociedad del riesgo", "sociedades postindustriales", "sociedad sitiada", entre otras nominaciones relevantes acuñadas por diversos autores. Estas expresiones manifiestan, de una u otra forma, que el cambio se ha constituido en una categoría central para el análisis de la experiencia personal y la organización de la sociedad actual.
\end{abstract}

Palabras clave: Cultura escolar, cambio cultural, jóvenes, sociedad.

\begin{abstract}
In broad terms, our reflection is to be found in the context of the social economic, political and cultural transformations that the contemporary societies live. These transformations have been outlined as one of the main phenomena in the beginning of XXI Century, and has been conceptualized in diverse ways: "society of the information", "risk society", "postindustrial societies", "siege society", among other outstanding expressions coined by diverse authors. These expressions declare in one way or another that the change has constituted in a central category for the analysis of the personal experience and the organization of the present society.
\end{abstract}

Key words: Scholastic culture, cultural change, young people, society.

* Walter Molina es Candidato a Doctor en Ciencias de la Educación de la Pontificia Universidad Católica de Chile.

Mario Sandoval es Doctor en Sociología de la Universidad Católica de Lovaina y Coordinador del Centro de Estudios en Juventud de la Universidad Católica Silva Henríquez, en Santiago de Chile. 


\section{Introducción}

En términos amplios, nuestra reflexión se ubica en el contexto de las transformaciones sociales, económicas, políticas y culturales que viven las sociedades contemporáneas. Estas transformaciones se han perfilado como uno de los principales fenómenos en el inicio del Siglo XXI y han sido conceptualizadas de diversos modos: "sociedad de la información", "sociedad del riesgo", "sociedades postindustriales", "sociedad sitiada", entre otras nominaciones relevantes acuñadas por diversos autores (Castell, 1997; Beck, 1995; Touraine, 1997; Bauman, 2004). Estas expresiones manifiestan, de una u otra forma, que el cambio se ha constituido en una categoría central para el análisis de la experiencia personal y la organización sociedad actual.

A nuestro juicio, estos cambios se insertan en el contexto de lo que algunos autores han descrito como un proceso de "mutación cultural" (Bajoit, 1995; Franssen et al., 1997; 2003; Sandoval, 1996; 2003), el cual expresaría el paso de un modelo cultural cuyo eje central es el valor del trabajo, la razón social y el progreso a un modelo cultural basado en la autorrealización autónoma de los actores sociales. Este proceso de mutación cultural ha generando nuevas tensiones y desafíos en diversos ámbitos de la sociedad, particularmente a nivel de las instituciones que tradicionalmente han ejecutado los roles de socialización e integración social: la familia, la escuela y el trabajo.

A nuestro juicio, las transformaciones que se están produciendo en las diversas esferas de la sociedad tienen un impacto directo en la (re)configuración de la cultura juvenil, la cultura escolar y el propio espacio escolar. En consecuencia, el presente artículo analiza algunos de los elementos de cambio que es posible observar tanto a nivel de la(s) cultura(s) juvenil(es) como en la(s) cultura(s) escolar(es) y su efecto en la (re)construcción simbólica del espacio escolar, específicamente del liceo. Finalmente, y a partir de la identificación de esos elementos de cambio, se proponen 
algunas acciones que permitan promover un acercamiento y una mejor articulación entre ambas culturas en el espacio escolar.

\section{Cultura escolar y cultura juvenil en Chile: algunos elementos de contexto}

En los últimos quince años, la enseñanza media chilena ha sido objeto de un conjunto de transformaciones: cambios curriculares, mejoramiento de infraestructura educativa, expansión de su cobertura, extensión de la jornada escolar y, recientemente el establecimiento de su gratuidad y obligatoriedad jurídica hasta los 21 años ${ }^{1}$. Estos cambios son resultado, en parte, de la implementación del programa de Mejoramiento de la Calidad y la Equidad de la Educación Media (Mineduc, 1994) el cual orientó sus objetivos, estrategias y recursos a producir una renovación de este nivel educativo, con la finalidad de adecuarlo a las transformaciones socioculturales en la configuración de las sociedades contemporáneas. A nuestro juicio, las transformaciones que se están produciendo en las diversas esferas de la sociedad tienen un impacto directo y significativo en la configuración de la cultura juvenil, la cultura escolar, produciendo con ello una resignificación del propio espacio escolar (el liceo).

Como es sabido, el componente joven del programa MECEMedia (Mineduc, 1994:12) implementó un conjunto de iniciativas tendientes a revalorizar la cultura juvenil al interior del liceo. Por consiguiente, se planteó como objetivo explícito producir una integración de la cultura juvenil a la cultura escolar con el fin de darle una mayor pertinencia cultural a los procesos educativos desarrollados en su interior. No obstante, luego de diez años de incorporación de dichas iniciativas, se observa la existencia de una serie de tensiones, desencuentros y conflictos no resueltos entre cultura juvenil y cultura escolar (Oyarzún, A; Irrazabal, R. Goicovic, I., y Reyes, L. 2001). Desde esta perspectiva, la articula-

1 Reforma constitucional del 7 /5/ 2003. 
ción entre ambas culturas sigue siendo una tarea pendiente para todo el sistema educativo y plantea, a nuestro juicio, la urgente necesidad de fomentar el reconocimiento mutuo entre los actores sociales estratégicos del proceso educativo: los jóvenes-alumnos y los profesores. Creemos que dicho reconocimiento es una de las bases que permitirían articular de mejor forma las culturas que co-existen al interior del espacio escolar del liceo y, con ello, contribuir a dar mayor pertinencia cultural a los procesos educativos que se desarrollan en la enseñanza media.

\section{Cultura y diversidad: una mirada desde la antropología cultural}

Una aproximación etimológica a esta noción nos revela que "cultura" procede del latín <colo> que significa cultivar. Por consiguiente, uno de sus significados más originarios nos remite al proceso por el cual se extraen las potencialidades de las semillas y de su domesticación. Y, por extensión (o por defecto), posteriormente se comienza a aplicar la noción de cultura al proceso de cultivo de algo: cultivo del espíritu, del estilo, los peces y las plantas. Así, la cultura se convierte en sinónimo de crianza o educación como e-ducare: sacar fuera, desarrollar las potencialidades latentes en el niño, entre otras asociaciones análogas (Ariño, 2000:14). A partir de esta raíz etimológica común, la cultura comienza a ser concebida como la capacidad humana de producir artefactos o bienes materiales y símbolos, es decir, en oposición a lo dado por la naturaleza; lo cultural se asocia a lo producido, lo cultivado en el marco de una determinada comunidad humana. De este modo, la noción de cultura viene a enfatizar la idea de que lo más característico de lo humano sería esta doble capacidad socioproductiva (tanto material como simbólica), la que permitiría al hombre mediatizar la relación con su entorno.

Como indica Arnold (1989), las definiciones de cultura en antropología generan una permanente fuente de controversias en lo que respecta a sus alcances, al contenido de la realidad que con él 
se invoca y principalmente a la determinación de sus elementos y el tipo de relaciones que entre estos se establecen. Sin embargo, existiría consenso con relación a que una cultura puede descomponerse, al menos, en tres niveles o esferas: material, social e ideacional. En el primer nivel, se incluyen todos los elementos tecnológicos, mecánicos y físicos que permiten la subsistencia material de las sociedades. Se consideran también los objetos de consumo y de valor evocativo simbólico de las comunidades humanas. El segundo nivel está referido a la manifestación y normatividad de todo tipo de relaciones interpersonales y grupales, es decir, roles, normas e instituciones sociales. Finalmente, en el nivel ideacional o ideológico, se incluyen todos los conocimientos, ideas, creencias, valores, lenguaje y, en general, todas las formas simbólicas aprendidas y compartidas en una sociedad (Arnold, 1989).

Estos niveles y sus respectivos elementos se pueden observar en el siguiente esquema:

Esquema 1: Componentes de la noción de cultura (Arnold, 1989)

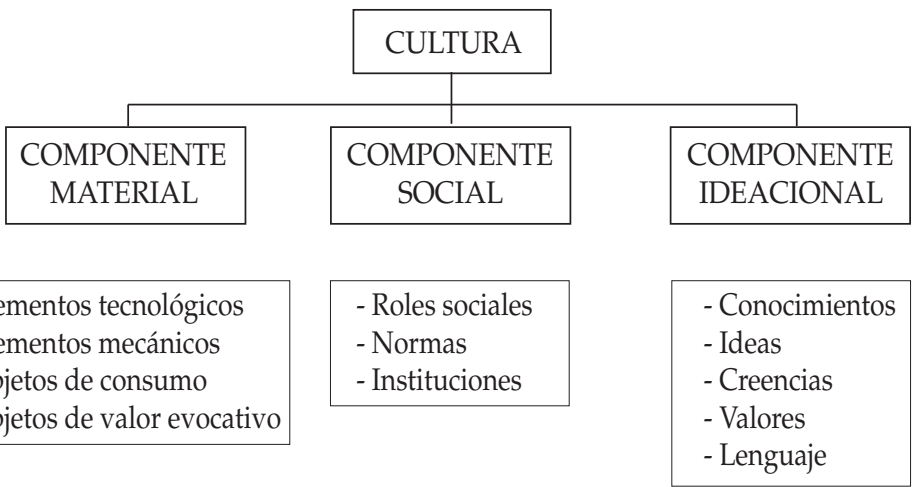

Como podemos observar en el cuadro anterior, la cultura se encuentra organizada sistemáticamente de determinada manera y, aunque solo puede ser descompuesta en sus elementos en forma analítica y no real, su esquematización permite una mejor comprensión del funcionamiento de los sistemas culturales concretos. Sin embargo, como señala Arnold (1989), entre los antro- 
pólogos existe consenso en que los niveles anteriormente indicados mantienen entre sí una relación de interdependencia, aun cuando, agrega el autor, no habría acuerdo con relación a cuál de estos tres componentes tiene preeminencia sobre los demás.

Ahora bien, ¿cuál es el sentido de esquematizar los componentes de la cultura? Su sentido reside en la necesidad de enriquecer nuestra mirada sobre el fenómeno cultural, especialmente en su manifestación al interior del espacio escolar que, siendo un espacio altamente institucionalizado, se tiende a sobredimensionar sus aspectos normativos y materiales, invisibilizando, con ello, su componente ideacional. Tradicionalmente, cuando se estudia, tanto la cultura escolar como la cultura juvenil se tiende a simplificar el análisis con el fin de destacar uno u otro componente cultural, lo cual no permite captar la complejidad interna de la cultura en estudio. En ocasiones, la cultura escolar o cultura juvenil aparecen como conceptos homogéneos, como si, en su interior, no existiese la heterogeneidad, diferenciación de elementos, relaciones entre ellos y, por sobre todo, la pluralidad de actores sociales que la co-construyen. Es por ello que el concepto de cultura nos permite acceder tanto a la diversidad interna de las comunidades humanas (su pluralidad de actores, de artefactos, de símbolos, actitudes, valores, entre otros aspectos relevantes) como a la diversidad externa, es decir, la pluralidad de culturas que co-existen y conviven en un mismo espacio social.

Finalmente, y dado que la escuela de nuestro tiempo se ha constituido en un espacio social cada vez más heterogéneo y complejo, creemos que el reconocimiento de esta diversidad y complejidad de base constituye un desafío permanente para todos los actores educativos. Es en este camino de (re)conocimiento donde la noción de cultura se presenta como un aporte significativo en pos de una mejor articulación entre la cultura escolar y la cultura juvenil. 


\section{Cultura y homogeneidad: una mirada desde la sociología de la cultura}

Así como desde la antropología la noción de cultura ha sido fecunda en develar los diferentes procesos, rostros y figuras de la diferenciación intra/inter comunidades humanas: diferencias étnicas, lingüísticas, económicas, etáreas, de género, entre otras, la sociología, a su vez, ha sido fértil en producir conceptos y teorías que, si bien dan cuenta de los procesos de diferenciación social, más bien tienden a enfatizar los elementos de continuidad, de estabilidad (aunque también de cambio) de los sistemas sociales. En consecuencia, sostenemos que el desarrollo teórico que la noción de cultura ha tenido al interior de la sociología ha estado más vinculado a explicar la problemática de la construcción del "orden social", de la "estructuración social"; que a destacar los elementos de diferenciación y diversidad social que se producen al interior de las sociedades. En este sentido, es sintomático, como indica Ariño (2000), que el exponente más sistemático de la teoría de la cultura común al interior de las ciencias sociales fuera Talcottt Parsons (1970) y su monumental obra, Teoría general de la acción.

Como es sabido, Parsons distingue cuatro sistemas constituyentes de la sociedad: social, de la personalidad, cultural y organismo conductual. Cada uno de estos sistemas se diferencia de los otros por su especialización en uno de los "imperativos funcionales" del sistema social global (adaptación, capacidad para alcanzar metas, integración y mantenimiento de patrones). De este modo, la cultura -o más precisamente los sistemas culturales- quedan definidos como un patrón de cultura cuyas partes se interrelacionan para formar sistemas de valor, de creencias y de símbolos expresivos estandarizados. Estos elementos culturales se institucionalizan en los sistemas sociales y se internalizan en los sistemas de personalidad. De este modo, "la cultura" guía al actor tanto en su orientación a fines como a la regulación normativa de los medios y las actividades expresivas que estos realizan en el marco 
de una sociedad concreta. En este contexto teórico, Parsons plantea que la realidad social se puede mirar desde tres perspectivas diferentes, como se muestra en el siguiente esquema:

Esquema 2: Las tres caras de la realidad social (Ariño, 2000:31)

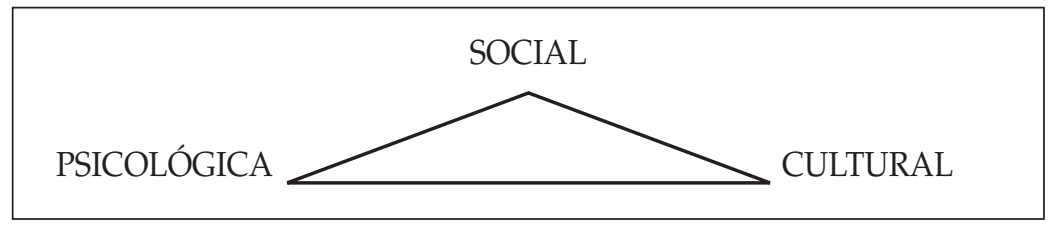

En el esquema anterior, la dimensión cultural no tiene existencia empírica en sí misma. Consiste en valores, creencias y símbolos inmanentes en la personalidad y en el sistema social, a los que accedemos por abstracción. Adicionalmente, el esquema nos muestra el modo en que, desde la sociología funcionalista clásica, se intenta explicar la estabilidad de los sistemas sociales, es decir, "en términos de la íntima interdependencia de los patrones culturales, la institucionalización y las necesidades personales" (Abercrombie et al., 1987:59).

En síntesis, la noción de cultura constituye una clave analítica indispensable, tanto para la antropología como para la sociología, que permite una aproximación al conocimiento de uno de los componentes esenciales de todo proceso de construcción de la realidad social. Y, si bien el concepto de cultura ha tenido desarrollos teóricos y metodológicos diferentes tanto en antropología como en sociología, estas diferencias, más que dificultar el análisis cultural, contribuyen a enriquecer la mirada sobre el fenómeno cultural en las sociedades contemporáneas.

\section{El espacio escolar: Actores en una encrucijada cultural}

Con la finalidad de precisar lo que entendemos por espacio escolar, nos apoyaremos en una definición que lo caracteriza de la siguiente manera: "Un espacio ecológico de cruces de cultu- 
ras cuya responsabilidad específica es la mediación reflexiva de aquellos influjos plurales que las diferentes culturas ejercen sobre las nuevas generaciones, para facilitar su desarrollo educativo" (Pérez Gómez, 1998:17). Consideramos que acotar el espacio escolar a partir de la metáfora: "espacio ecológico de cruce de culturas" nos permite ampliar la mirada de la escuela o el liceo más allá de su finalidad explícita de reproducción social. En esta aproximación teórica, la escuela ya no se concibe solo como un espacio físico-institucional altamente normativizado o como un instrumento de transmisión y reproducción cultural, sino que, por el contrario, la escuela se define como un "espacio ecológico relacional" donde se interceptan e interactúan diferentes culturas y sujetos, los que, además, son (co)constructores de la totalidad de los procesos educativos que ocurren en su interior.

En este sentido, Pérez Gómez (1998) propone una interesante taxonomía de las diversas culturas que interactúan en el espacio escolar. Sin establecer jerarquías entre ellas, el autor las define como:

a) Cultura crítica: hace referencia a las disciplinas científicas que nutren de diversa forma el proceso educativo.

b) Cultura social: expresa los valores y normas dominantes en una sociedad, lo cual produce un influjo tanto en profesores como alumnos.

c) Cultura institucional: hace referencia a los ritos, normas y valores característicos de la cultura escolar (en sentido restrictivo).

d) Cultura experiencial: sería la cultura propia de los diversos sujetos que interactúan en el espacio escolar, es decir, la cultura que portan los jóvenes estudiantes, los profesores, los directivos, los apoderados, entre otros actores del proceso educativo.

e) Cultura académica: se manifiesta en el curriculum escolar 
que es, por definición, un mecanismo de selección de los elementos culturales (saberes, normas, valores, etc.) que se ponen en juego con una finalidad educativa al interior del espacio escolar.

En síntesis, la identificación de cinco culturas en un mismo espacio escolar nos permite comprender que la diversidad cultural no es solo un asunto referido a la existencia de diversas culturas a nivel macrosocial o fuera del espacio educativo formal (Cultura Mapuche, Cultura Aymará, etc.) sino, por el contrario, esta diversidad es un rasgo característico del espacio escolar. Adicionalmente, este enfoque permite visibilizar los diversos actores del proceso educativo que interactúan cotidianamente en este espacio; esto es: profesores, jóvenes estudiantes, padres, apoderados y todos aquellos interesados en influir en la educación de (nos)otros.

A continuación, presentamos un esquema donde se pueden observar las diversas culturas que se interceptan en el espacio escolar:

Esquema 3: Culturas que se interceptan en el espacio escolar (Pérez Gómez, 1998)

ESPACIO ESCOLAR

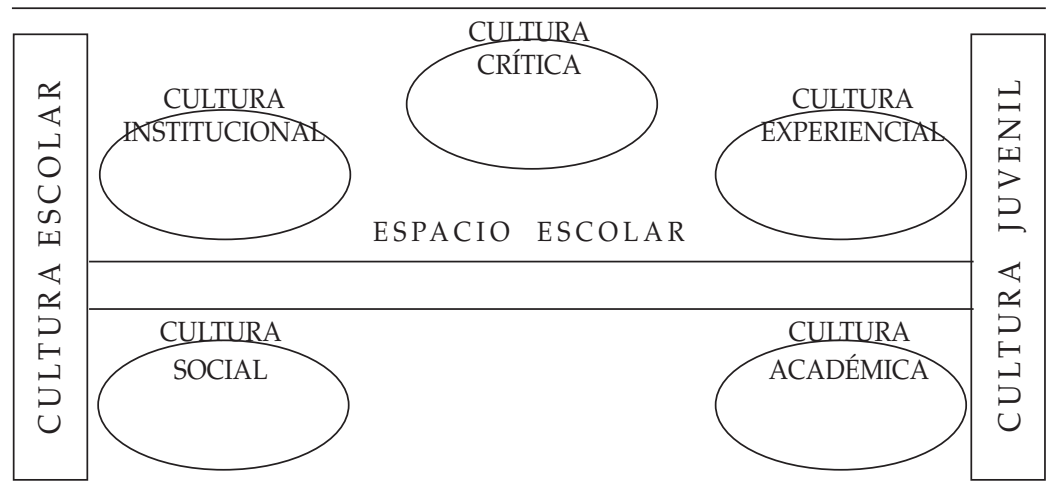

Como podemos observar, los actores educativos están ex- 
puestos al influjo permanente de diversas culturas y, por lo tanto, no solo podemos hacer nuestro análisis a partir de una relación dicotómica entre cultura escolar y cultura juvenil sino, además, debemos reconocer la existencia de una pluralidad de culturas que cohabitan en el espacio escolar. Estas culturas en interacción cotidiana, tanto al interior del aula como fuera de ella, producen influjos recíprocos en los actores que entran en contacto en el proceso educativo y, por lo tanto, requieren de un reconocimiento genuino de la existencia de esta pluralidad cultural como condición para su articulación al interior del espacio escolar.

\section{Aproximación conceptual a la cultura escolar y la cultura juvenil}

La cultura escolar ha sido abordada desde diversas aproximaciones conceptuales que difieren entre sí, tanto por el contenido de la realidad que con ella se invoca como por la determinación de los elementos que la componen. Esta multiplicidad de enfoques va desde definir operativamente el concepto como: "Dinámicas que se generan al interior del establecimiento, pero fuera de la sala de clases" (Errázuriz, et al., 1994), hasta definiciones más generales y abarcadoras como: "El conjunto de conocimientos, estados anímicos, acciones y nivel de desarrollo alcanzado por una comunidad educativa" (Martínez- Otero, 2003). Desde nuestra propia perspectiva, y teniendo como base las precisiones conceptuales anteriormente presentadas, la cultura escolar se considerará como el resultado de una construcción social contingente a las condiciones materiales, sociales y simbólicas que dominan en un tiempo y en un espacio determinado, esto es: "Conjunto de significados, expectativas y comportamientos compartidos por un determinado grupo social, que facilitan y ordenan, limitan y potencian los intercambios sociales, las producciones simbólicas y materiales y las realizaciones individuales y colectivas dentro de un marco espacial y temporal determinado" (Pérez Gómez,1998:16).

En esta visión, como vemos, se integran los aspectos mate- 
riales, sociales e ideacionales que conforman genéricamente una cultura. Esta perspectiva nos permitirá, en lo sucesivo, intentar describir y analizar los diversos elementos y relaciones involucradas en la configuración tanto de la cultura escolar como de la cultura juvenil.

Para especificar los contenidos de nuestra propia definición, presentamos, a continuación, un esquema donde se pueden observar los componentes y elementos que estructuran la cultura escolar:

\section{Esquema 4: Componentes de la noción de cultura escolar}

\section{CULTURA ESCOLAR}

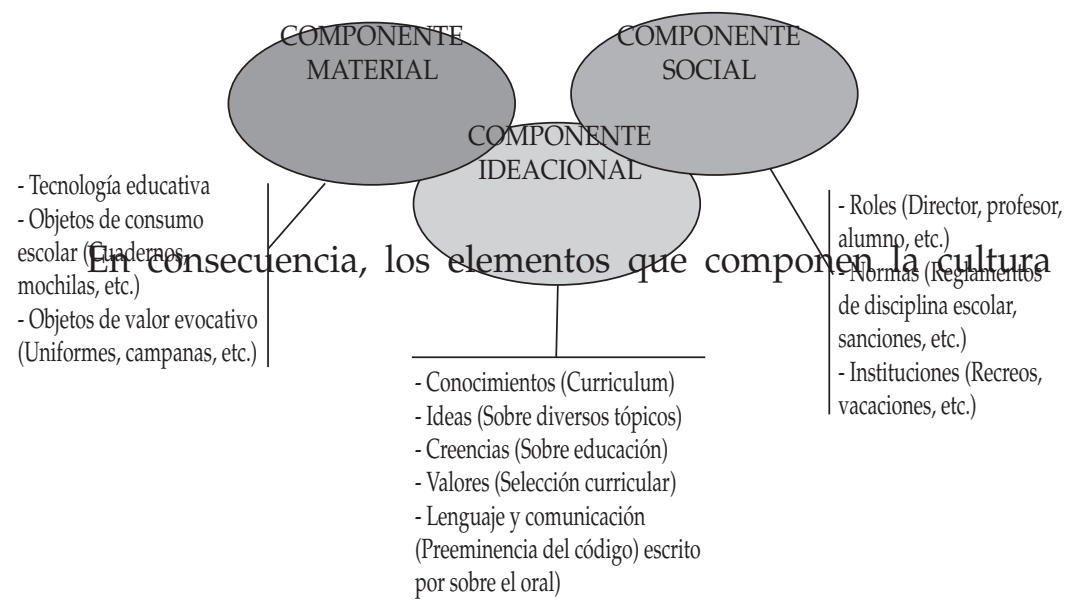

escolar serían: normas, mitos, signos, ritos, lenguaje y comunicación, valores y supuestos básicos implicados en la práctica educativa (teorías implícitas de los profesores, representaciones sociales sobre diversos tópicos de lo educativo, etc.) Tanto a partir de la definición como de la enumeración de elementos, se puede observar que la cultura escolar tiene una doble funcionalidad: establecer los límites de la institución y proporcionar elementos de identidad y sentido de pertenencia a los miembros que la com- 
ponen.

Por otra parte, la definición y caracterización de la cultura juvenil requiere de un análisis previo de la noción de juventud. Al respecto, existen diversas perspectivas de análisis del fenómeno juvenil: "La juventud como una simple categoría etárea (1529 años); la juventud como etapa de maduración psicológica; y, finalmente, la juventud como una subcultura" (Sandoval, 2002: 159-164). En el marco de esta última concepción, consideraremos la noción de cultura (s) juvenil (es) como: "La manera en que las experiencias sociales de los jóvenes son expresadas colectivamente mediante la construcción de estilos de vida distintivos, localizados en el tiempo libre o en espacios intersticiales de la vida institucional" (Feixa, 1998: 84). En consecuencia, la condición juvenil, tal como lo señala este autor, estaría dada por un estilo de vida que se constituye en uno de los elementos distintivos de las culturas juveniles y que el mismo autor define como: "manifestaciones simbólicas propias, expresadas en un conjunto más o menos coherente de elementos materiales e inmateriales que los jóvenes consideran representativos de su identidad como grupo" (Feixa, 1998: 85).

En este sentido, podemos considerar a las culturas juveniles como el resultado de un doble proceso de diferenciación: en primer lugar, del mundo adulto y, luego, establecimiento de "fronteras interiores" entre sus propios pares. Ambos procesos contribuyen a establecer la identidad de los sujetos: tanto en el ámbito individual como en el colectivo. A continuación, presentamos un esquema que intenta sintetizar los principales elementos de la cultura juvenil:

Esquema 5: Componentes de la noción de cultura juvenil 


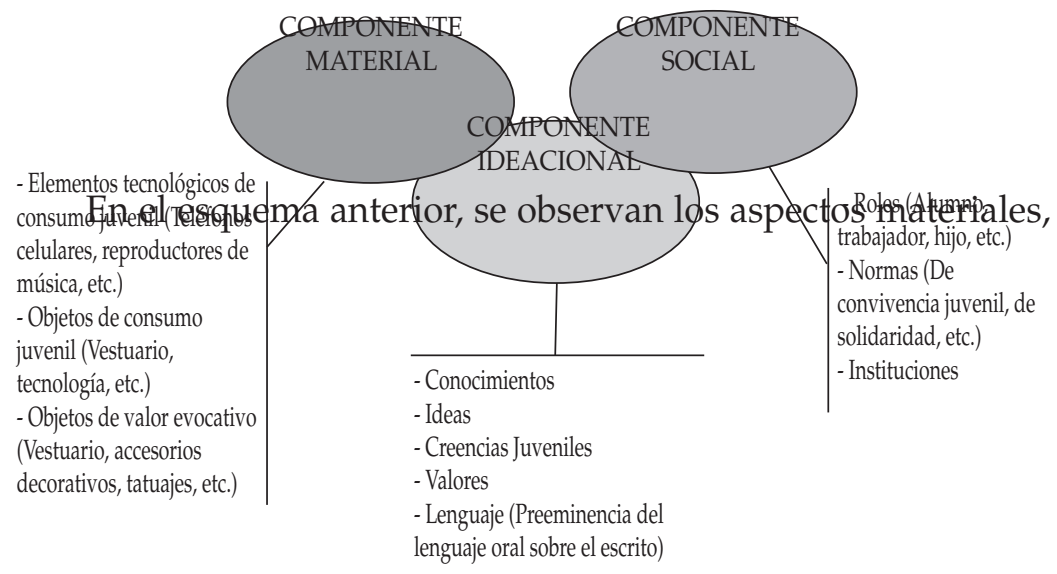

sociales e ideacionales que componen toda cultura y que, en el caso de la cultura juvenil, revelan un complejo proceso de resignificaciones simbólicas que hacen los jóvenes de dichos elementos (artefactos culturales de consumo juvenil, roles y normas, etc.).

Como una manera de sintetizar los principales conceptos revisados en este artículo, se presenta, a continuación, un esquema donde se muestran las diferentes culturas que interactúan al interior del espacio escolar y cómo ellas enmarcan la relación entre lo que hemos llamado cultura escolar y cultura juvenil. Esquemáticamente, se presenta del siguiente modo:

Esquema 6: Articulación de la cultura escolar y la cultura juvenil en el espacio escolar

ESPACIO ESCOLAR

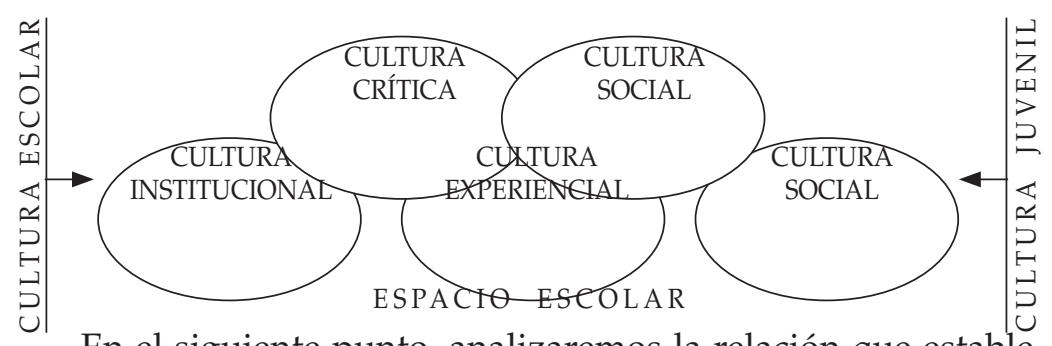

En el siguiente punto, analizaremos la relación que estable- 
cen en el espacio escolar la cultura escolar y la cultura juvenil. En este marco, y a partir de las nociones teóricas revisadas, interpretaremos algunas de las diversas manifestaciones de esta interacción cultural al interior del espacio escolar, enfatizando para ello la dinámica de encuentros y desencuentros que se producen.

\section{Cultura juvenil y cultura escolar: (des)encuentros al interior del espacio escolar}

La relación entre cultura escolar y cultura juvenil ha sido presentada en términos de una relación conflictiva, la cual se produciría por una tendencia inherente que lleva a la escuela/liceo, en tanto institución social reproductora de un orden social, a cerrarse respecto de las experiencias de los jóvenes en el espacio exterior al liceo (Cerda, Assaél, Ceballos y Sepúlveda, 2000: 21).

Desde esta perspectiva, la escuela/liceo manifestaría una tendencia a la homogeneización de los jóvenes estudiantes en la categoría de "alumnos", todos iguales, dentro de un mismo régimen de enseñanza y disciplina escolar. De este modo, las significaciones y experiencias que del "mundo exterior" extraen los sujetos son consideradas, por el sistema educativo, como secundarias y/o perjudiciales, pues limitan la igualación que se produce con la escolarización de los jóvenes. Los autores mencionados concluyen que el reconocimiento de intereses, de expectativas, de experiencias, es un aspecto que las instituciones escolares no han incorporado aún. En esta coyuntura, la relación entre ambas culturas es de incomunicación, donde las prácticas escolares y los contenidos curriculares no se condicen con la "cultura experiencial" de los jóvenes-alumnos, generando con ello una fuente permanente de tensiones y desencuentros entre cultura escolar y cultura juvenil.

Desde esta perspectiva, el problema fundamental en la relación entre cultura juvenil y cultura escolar consistiría en que esta última no ha logrado reconocer que la educación, particularmente la enseñanza media, es la educación de los jóvenes y para los 
jóvenes. En consecuencia, los problemas de articulación o desarticulación no se producirían entre jóvenes, sino que entre estos y una cultura escolar que no les reconoce su condición de tales, sino que, por el contrario, "tiende a reglamentar y a normar sus conductas de manera rígida y, a veces, de manera hasta abusiva" (Oyarzún, et al., 2001:15).

Podemos inferir, por tanto, que una fuente importante de tensiones entre ambas culturas radicaría en el tipo de relaciones sociales que se establecen entre jóvenes y profesores al interior del espacio escolar. Estas relaciones se caracterizarían por el temor, la desconfianza y el miedo. Como señala Duarte (2002), las relaciones entre el mundo adulto (los profesores) y el mundo joven (los alumnos) están mediadas por una serie de máscaras y corazas que impiden la espontaneidad y la autenticidad entre ellas, siendo finalmente estas máscaras las que definen los estilos de relaciones que se materializan a través de roles, estatus, atribuciones de poder y otras formas concretas de presentarse ante el mundo. Al respecto, se señala lo siguiente:

“El profesor Luis y la alumna Sara no se relacionan en tanto personas, sino a partir y mediante los roles que cada cual juega en el espacio escolar. De esta manera, las posibilidades de acercamiento y vinculación profunda estarán dadas solo si son capaces de abrirse y mostrarse con autenticidad en la relación" (Duarte, 2002:110).

Al respecto, podemos señalar que la relación pedagógico-formativa entre educador y educando se ha descrito desde diversas perspectivas tomando en consideración dos características básicas: en primer lugar, se trata de una relación social, pues están implicados al menos dos actores sociales y, en segundo lugar, se trata de una relación social asimétrica, porque siempre está claro quién educa y quién es educado. En consecuencia, no se trata de una relación social igualitaria. Sin embargo, creemos que la interpretación y el desempeño de los roles en el ámbito educativo 
debiera tender a flexibilizarse y a adecuarse a las actuales tendencias de cambio que se observan en nuestra sociedad.

Como una forma de sistematizar los encuentros y desencuentros entre la cultura escolar y la cultura juvenil, proponemos analizar algunas tendencias de cambio sociocultural que, a nuestro juicio, son ilustrativas de la tensión entre ambas culturas.

Un primer foco de tensión se da en el seno del componente social de la cultura y gira la tensión en torno a la emergencia de nuevas formas de relación de los sujetos con la autoridad y el poder (y sus figuras tradicionales). En este sentido, y en la búsqueda de una mejor articulación entre la cultura escolar y la cultura juvenil, podemos observar que los aspectos sociales de la cultura (dimensión normativa, asignación de roles, distribución del poder, etc.) tienen una importancia estratégica para el logro de dicha articulación. Por ejemplo, mientras que en la cultura escolar los roles tienden a ser desempeñados de forma rígida y apegada a la tradición y acompañados de normas que refuerzan lo reglamentario, lo disciplinario (lo punitivo, a veces) y la dimensión instrumental de la acción educativa. En la cultura juvenil, en cambio, los roles y estatus tradicionales (alumno, trabajador, hijo, etc.) son desempeñados en forma más flexible y resignificados de diversas maneras dando origen, con ello, a nuevas formas de relación de los jóvenes con la normatividad, las figuras de autoridad, las oportunidades de acceso a diversos bienes sociales, las expectativas de éxito social, etc. Con ello, consideramos que un desempeño rígido de los roles educativos genera una tendencia a entrar en conflicto con la normatividad escolar y sus figuras de autoridad educativa (profesores, directores, inspectores, etc.).

Un segundo foco de tensión gira en torno a la emergencia de nuevas formas de relación de los actores sociales (particularmente los jóvenes) con la información y el conocimiento (y sus instrumentos más visibles: la tecnología y los sistemas educativos). Lo anterior nos remite al componente social de una cultura. 
Un tercer foco de tensión entre cultura juvenil y cultura escolar está referido a los conocimientos, ideas, creencias, valores, lenguaje y comunicación que emergen en el seno de las culturas juveniles en la actualidad y que no son reconocidos como formas de expresión socialmente legítimos por la cultura escolar. Estos rasgos emergentes de la cultura juvenil, que nos remiten directamente al nivel ideacional de las culturas, conformarían los nuevos "universales culturales" (Margulis y Urresti, 1998) de la condición juvenil en la época contemporánea. Un ejemplo de estos rasgos sui generis serían: predominio de la imagen por sobre lo discursivo (oral o escrito), predominio del presente como dimensión temporal dominante en desmedro del futuro, entre otros aspectos relevantes.

Finalmente, podemos sostener que la noción de cultura brinda múltiples potencialidades para el análisis educativo. El enfoque cultural no supone un simple cambio de denominaciones, sino "Una nueva perspectiva de análisis de lo que realmente ocurre en la escuela y de los efectos que tienen la(s) cultura(s) en los pensamientos, sentimientos y conductas de estudiantes y profesores" (Pérez Gómez 1998:16). En este sentido, lograr una comprensión cultural del fenómeno educativo requiere descender a los intercambios (tanto implícitos como explícitos) de significados que se producen en los momentos y en las situaciones más diversas e inadvertidas de la vida cotidiana en el ámbito de la escuela.

\section{Reflexiones finales}

Más que conclusiones definitivas, se presenta una serie de reflexiones que permitan estimular el debate educativo y explorar nuevos caminos en pos de una mejor articulación entre la cultura escolar y la cultura juvenil.

En primer lugar, queremos insistir en la necesidad de un reconocimiento de la diversidad cultural realmente existente al interior del espacio escolar: cultura crítica, cultura social, cultura institucional, cultura académica y cultura experiencial. Este 
reconocimiento es uno de los principales desafíos de la escuela contemporánea.

En segundo lugar, sostenemos que uno de los principales obstáculos para producir una real articulación entre cultura escolar y cultura juvenil reside en el hecho de que, en el espacio escolar, solo se reconoce una dimensión de la condición existencial de los jóvenes: su condición de alumno. De tal modo que, al enfatizar esta dimensión, se tiende a dejar fuera del proceso educativo aspectos clave de la formación de los jóvenes (y de su cultura), es decir, sus intereses, sus necesidades, los elementos estéticos propios, entre otros aspectos fundamentales de su proceso de construcción de identidad individual y colectiva.

Una tercera reflexión que permite (re)pensar en una (re)articulación entre cultura(s) juvenil(es) y cultura(s) escolar(es) tiene que ver con el hecho de que ambas culturas se encuentran inmersas en la actualidad en una dinámica de cambios sociales que está generando profundas transformaciones en el ámbito de la subjetividad de los individuos, la estructura y función de las instituciones educativas, emergiendo con ello nuevas formas de relación entre individuo, educación y sociedad.

En cuarto lugar, podemos señalar que la importancia otorgada al análisis del fenómeno cultural juvenil en Chile se manifiesta en una variedad de estudios e investigaciones (Duarte, 1994; Sandoval, 2002; Zarzuri y Ganter, 2002; Guell, 2003). Potencialmente, estos estudios otorgan a los diversos actores del sistema educativo la posibilidad de acceder a una comprensión más profunda de la(s) cultura(s) juvenil(es) que convergen en el espacio escolar. En relación con la cultura escolar, se destaca una amplia gama de autores y estudios (Edwards et al., 1995; Cerda et al., 2000; Oyarzún et al., 2001; Baeza, 2001) en los cuales se encuentra una riqueza conceptual y analítica que proporcionan nuevas pistas y orientaciones que pueden contribuir significativamente a una mejor articulación entre cultura(s) juvenil(es) y culturas escolar(es). 
Finalmente, sostenemos que el "cruce de culturas al interior del espacio escolar" (Pérez Gómez, 1998) debe tener como eje el reconocimiento de la "cultura experiencial" de los actores estratégicos del sistema educativo: jóvenes y profesores. De modo tal que la especificidad de cada cultura que se intercepta no se constituya en un obstáculo para su articulación, sino más bien en una oportunidad para enriquecer la cultura escolar y la propia "cultura experiencial" de los sujetos concernidos en la educación.

\section{Bibliografía consultada}

Ariño, A. (2000). Sociología de la cultura. La constitución simbólica de la sociedad. España: Ariel.

(2003). "Diversidad cultural". En: Diccionario de la Solidaridad (I). Valencia: Fundación de la Solidaridad y el Voluntariado de la Comunidad Valenciana.

Baeza, J. (2001). El oficio de ser alumno, en jóvenes de liceo de sector popular. Santiago: Universidad Católica Raúl Silva Henríquez.

Cerda, A. M.; Assaél, Y; Ceballos, F. \& Sepúlveda, R. (2000). Joven y alumno: ¿Conflicto de identidad? Un estudio etnográfico en los liceos de sectores populares. Santiago: LOM.

Duarte, C. (2002). "Mundos jóvenes, mundos adultos: lo generacional y la reconstrucción de los puentes rotos en el liceo. Una mirada desde la convivencia escolar". Revista Última Década.16.9. 5113.

Edwards, V. (1995). "El liceo por dentro. Estudio etnográfico sobre prácticas de trabajo en Educación Media". Santiago de Chile: MINEDUC.

Errázuriz, M.; González, R.; Martinic, S.; Manzi, J.; Scharager, J.; Swope, J. \& Urzúa, P. (1994). Demandas sociales a la educación media. Santiago: Ministerio de Educación de Chile.

Feixa, C. (1998). "La ciudad invisible. Territorios de las culturas juveniles". En M. Margulis \& M.Urresti (Coord.). Viviendo a toda: jóvenes, territorios culturales y nuevas sensibilidades. Bogotá: Siglo del hombre editores.

Guell, P. (2003). Transformaciones culturales e identidad juvenil en Chile. Santiago, Editorial: PNUD. 
Margulis, M. y Urresti, M. (1998). "La condición social de la condición de juventud". En M. Margulis \& M. Urresti (Coord.). Viviendo a toda: jóvenes, territorios culturales y nuevas sensibilidades. Bogotá: Siglo del hombre editores.

Martínez-Otero, V. (2003). Cultura escolar y mejora de la educación. Madrid: Complutense.

MINEDUC (1994). Fundamentos, estrategias y componentes del Programa de Mejoramiento de la Calidad y Equidad de la Educación Media. Santiago de Chile: Ministerio de Educación.

Oyarzún, A.; Goicovic, I.; Irrazabal, R. \& Reyes, L. (2001). Entre jóvenes reproductores y jóvenes co-constructores: sentidos de la integración en la cultura escolar. Santiago: Ministerio de Educación.

Pérez Gómez, A. (1998). La cultura escolar en la sociedad neoliberal. Madrid: Morata.

Sandoval, M. (2002). Jóvenes en el siglo XXI. Sujetos y Actores en una sociedad en cambio. Santiago de Chile: Ediciones Universidad Cardenal Silva Henríquez.

Zarzuri, R. y Ganter, R. (2002). Culturas Juveniles, narrativas minoritarias y estéticas del descontento. Santiago: UCSH. 